\title{
Les Trois Mouvements perpétuels de Poulenc : cycle en blanc et noir (1918)
}

\section{Sylvie Douche}

Résumé : Le jeune Poulenc s'essaie à un hommage polyphonique dans ces trois pièces pianistiques relevant de la cyclicité et qui contribueront à le faire connaître. Il s'y montre maître des contrastes et manipule les structures harmoniques, phraséologiques, dynamiques, scalaires, en de larges oppositions ou superpositions à l'égal d'un peintre. L'analyse mettra en évidence les liens entre la Sonatine (Ravel) et les Mouvements perpétuels, dans un esprit redevable à Satie. En même temps, le compositeur tente de se démarquer de la figure tutélaire debussyste. En tout cela, Poulenc s'avère éminemment français.

Resumo: O jovem Poulenc se lança numa homenagem polifônia nessas três peças para piano reveladoras de uma ciclicidade e que contribuirão para torna-lo concehcido. Ele se revela metre dos contrastes e manipula as estruturas harmônicas, fraseológias, dinâmias, esalares, em amplas oposições ou superposições como um pintor. A análise tornará evidente as ligações entre a Sonatine (Ravel) e os Mouvements perpétue/s, num espírio que ele deve à Satie. Ao mesmo tempo, o compositor tenta se distanciar da figura tutelar de Debussy. Em tudo isto, Poulenc se confirma eminentemente françês.

«Après tous les brouillards impressionnistes, cet art simple, clair, renouant la tradition de Scarlatti et de Mozart ne serait-il pas la prochaine phase de notre musique? En tous cas, j'ai le souvenir d'avoir senti, ce jour-là, que la place de Poulenc serait grande et que son œuvre compterait ${ }^{1} »$. Ainsi conclut Darius Milhaud évoquant sa première rencontre musicale avec le jeune compositeur encore mobilisé. II venait d'assister chez René Chalupt, à une audition des Mouvements perpétuels (et du Bestiaire). Les trois courtes pièces pour piano sont datées de décembre 1918 et leur première audition a lieu début 1919 salle Huyghens par Ricardo Viñes lors d'un concert de la Lyre et Palette?

\footnotetext{
1 Darius Milhaud, Ma vie heureuse, Paris, Belfond, 1987, p. 82-83.

2 Le manuscrit est conservé à la New York Library (Lincoln Center).
} 
Poulenc tenait absolument à ce que ce fût Viñes qui en fût le créateur et il le lui fit rapidement savoir ${ }^{3}$ connaissant ce pianiste depuis 1914, auprès duquel il prit des cours jusqu'en 19174. C'est Viñes qui va l'introduire auprès de Gédalge, Satie, Albéniz ou Ravel. Cette même année 1917, Poulenc conçoit son premier triptyque pour piano, les trois Pastorales. Le 17 janvier 1918 , il est mobilisé, mais travaille beaucoup et nombre de compositions de cette époque le projettent sur la scène musicale ${ }^{5}$ : les années 1917-1919 sont des années charnières où le jeune compositeur se découvre véritablement lui-même.

Les Trois mouvements perpétuels sont dédiés à son amie Valentine Gross ${ }^{6}$ avec ces mots : " Pour vous ma chère amie, ces trois pièces minuscules et la profonde affection de votre fidèle Francis Poulenc ». Minuscules, ces pièces le sont certes par leur brièveté. Elles renouvellent cependant le genre du moto perpetuo - on ne peut s'empêcher de songer à un clin d'œil à l'ami Erik Satie - et elles ont cela d'étonnant qu'elles utilisent le clavier d'ivoire et d'ébène comme une palette à deux couleurs principales. II n'est que de considérer les nombreux para-textes qui jalonnent la partition pour comprendre qu'un jeu d'oppositions et de contrastes fonde - à tout niveau - ce parcours dont on verra qu'il est cyclique. Si la progression agogique est contrastée - d'un tempo «assez modéré » (144 à la noire) à «Alerte » (138 à la noire) en passant par un «Très modéré » central (92 à la noire) - l'on

3 Il l'avait rencontré grâce à l'entremise de Geneviève Sienkiewicz en 1914 et il lui écrit: "je viens vous demander de les créer [les Trois Mouvements perpétue/s] le samedi 9 février au soir, à Peinture et Musique, rue Huyghens » (lettre de Pont-sur-Seine, [janvier 1919], dans Myriam Chimènes (éd.), Francis Poulenc, Correspondance (1910-1963), Paris, Fayard, 1994, p. 84).

4 C'est cependant auprès de sa mère (Jenny) qu'il avait débuté le piano, elle qui jouait Mozart, Chopin, Schubert et les romances alors en vogue.

5 Songeons à Toréador (sur Cocteau), ses Sonates pour quatre mains et à deux clarinettes, ainsi que son Bestiaire ou Cortège d'Orphée (sur Apollinaire), voir plus loin.

6 Valentine Gross (1887-1968) est illustratrice et peintre décoratrice de théâtre. Elle épouse Jean Hugo en 1919 dont elle divorce dix ans plus tard avant de devenir la compagne d'André Breton. Très proche de Poulenc, elle est également la dédicataire de deux mélodies : "Il la prend dans ses bras » (Cinq Poèmes, Paul Éluard) et « Une roulotte couverte en tuiles» (Tel jour, telle nuit, Paul Éluard). 
constate que l'on reste dans un tempo moyen, de faible amplitude?. II en va de même souvent pour les contrastes de dynamique. Le tableau suivant liste l'ensemble des indications tout en les classant ${ }^{8}$.

Figure 1 : indications portées sur la partition des Mouvements perpétue/s de Poulenc

\begin{tabular}{|c|c|c|c|}
\hline & $\begin{array}{l}\text { Extrême } \\
\text { supérieure }\end{array}$ & $\begin{array}{l}\text { Position } \\
\text { médiane }\end{array}$ & $\begin{array}{l}\text { Extrême } \\
\text { inférieure }\end{array}$ \\
\hline \multirow{3}{*}{ Didascalies } & En dehors $(\mathrm{I} / 5)$ & $\begin{array}{c}\text { En général } \\
\text { sans nuances } \\
(\mathrm{I} / 1)\end{array}$ & $\begin{array}{c}\text { Doucement } \\
\text { timbré (I/12) } \\
\text { Incolore et } \\
\text { toujours piano } \\
(1 / 14)\end{array}$ \\
\hline & $\begin{array}{c}\text { Très chanté } \\
\text { (II/7) }\end{array}$ & $\begin{array}{c}\text { Indifférent } \\
(\mathrm{II} / 1)\end{array}$ & $\begin{array}{l}\text { Légèrement } \\
\text { timbré (II/ } 11)\end{array}$ \\
\hline & $\begin{array}{c}\text { Le chant en } \\
\text { dehors (III/8) } \\
\text { Avec charme } \\
\text { (III/28) } \\
\text { En dehors } \\
\text { (III/51) } \\
\text { Laisser vibrer } \\
\text { (III/57) }\end{array}$ & $\begin{array}{c}\text { Sans dureté } \\
\text { (III/4) } \\
\text { Gris (III/40) } \\
\text { Uniforme } \\
\text { (III/20) } \\
\text { Flou (III/47) }\end{array}$ & $\begin{array}{c}\text { Ralentir en } \\
\text { s'effaçant (III/57) }\end{array}$ \\
\hline
\end{tabular}

7 Poulenc met en garde ses interprètes contre la fantaisie agogique : «Si les pianistes faisaient confiance à mes mouvements métronomiques, très soigneusement établis, bien des malheurs seraient évités » (Francis Poulenc, Entretiens avec Claude Rostand, Paris, R. Julliard, 1954, p. 35).

8 Entre parenthèses : le premier chiffre (romain) est affecté au numéro de la pièce concernée, suit le numéro de mesures. 


\begin{tabular}{|c|c|c|c|}
\hline \multirow{3}{*}{$\begin{array}{l}\text { Indications } \\
\text { dynamiques }\end{array}$} & $F(\mathrm{I} / 10)$ & $\begin{array}{c}M f(I / 5) \\
M f(I / 22)\end{array}$ & $\begin{array}{c}P(\mathrm{I} / 1-8-12) \\
P(\mathrm{I} / 20) \\
P P(\mathrm{I} / 24)\end{array}$ \\
\hline & & $\begin{array}{c}M f(\mathrm{II} / 7) \\
\text { Clair mais } \\
\text { piano }(\mathrm{II} / 9)\end{array}$ & $\begin{array}{c}P(\mathrm{II} / 1-5) \\
P P(\mathrm{II} / 11) \\
P(\mathrm{II} / 13) \\
P P P(\mathrm{II} / 14)\end{array}$ \\
\hline & $\begin{array}{c}F(I I I / 1) \\
F F(I I I / 4) \\
F(I I I / 6) \\
F(I I I / 16) \\
F(I I I / 37)\end{array}$ & $\begin{array}{c}M f(\mathrm{III} / 8) \\
M f(\mathrm{III} / 18) \\
M f(\mathrm{III} / 24-28)\end{array}$ & $\begin{array}{c}P(\mathrm{III} / 7) \\
P(\mathrm{III} / 20-26) \\
P P(\mathrm{III} / 40) \\
P(\mathrm{III} / 47-53) \\
P P P(\mathrm{III} / 55-57)\end{array}$ \\
\hline \multirow{3}{*}{$\begin{array}{l}\text { Indications de } \\
\text { pédale }\end{array}$} & & $\begin{array}{c}\text { Péd. tenue } \\
\text { toute la } \\
\text { mesure finale } \\
(1 / 24)\end{array}$ & \\
\hline & & $\begin{array}{c}\text { Deux pédales } \\
(\mathrm{II} / 11)\end{array}$ & \\
\hline & & $\begin{array}{l}\text { Les deux } \\
\text { pédales } \\
\text { (III/40) } \\
\text { Les deux } \\
\text { pédales } \\
\text { (III/47) }\end{array}$ & \\
\hline
\end{tabular}

Notre article envisagera donc ces trois courtes pièces sous trois éclairages différents. D'une part en s'interrogeant sur la construction mélodico-harmonique qui fera nettement apparaître ce balancier de contrastes que (faute de mieux) nous avons dénommé «en blanc et noir» (entendre le plus souvent «le blanc avec le noir»), d'autre part en 
examinant l'organisation formelle globale et le travail motivique entrepris, redevable à l'ami admiré qu'est Maurice Ravel.

\section{En blanc et noir}

L'on a coutume de voir dans ces Mouvements perpétuels un retour à une simplicité tournant le dos aux emphases passées. Le premier motif travaillé, à trois voix, est ainsi inscrit dans une gamme en mode de SOL sur $f a$ (défective et sous sa forme descendante) en main droite, accompagné en main gauche par un mouvement pendulaire $\hat{1}-\hat{5}$ Les deux voix extrêmes sont contrepointées par une partie intermédiaire en sixtes parallèles posées sur $\hat{5}-\hat{6}-\hat{7}-\hat{6}-\hat{5}$

Figure 2 : Francis Poulenc, Mouvements perpétue/s (I), mes. 1-2

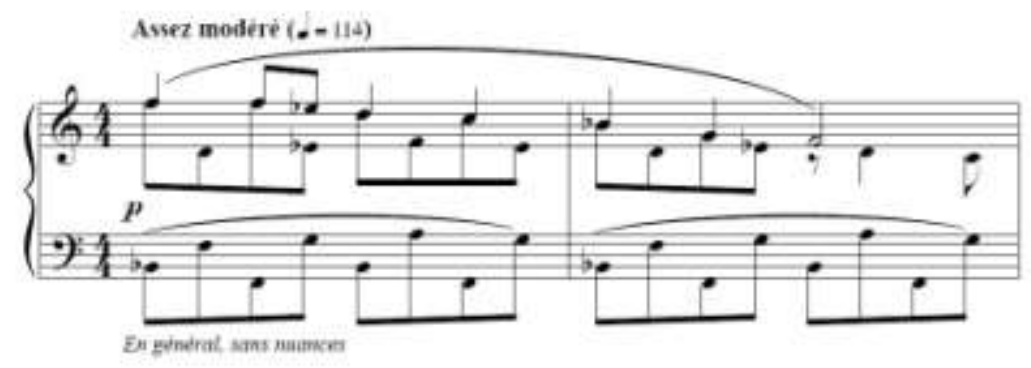

À aucun moment la main gauche ne varie dans ce premier Mouvement. La main droite, en revanche transpose ce premier motif ${ }^{9}$ et en propose trois autres. Celui que nous appelons $b$ est un mouvement conjoint inscrit dans le premier pentacorde de $D O$ majeur (dominante de la dominante en $S / b$ ).

9 Sur SOLb mesures 10-11, introduisant également un mordant au troisième temps et dans une nuance forte qui jure sur la demi-teinte générale de part et d'autre, la tonalité de $S O L b$ simulant un relief de polytonalité. 
Figure 3 : Francis Poulenc, Mouvements perpétuels (I), mes. 5-7

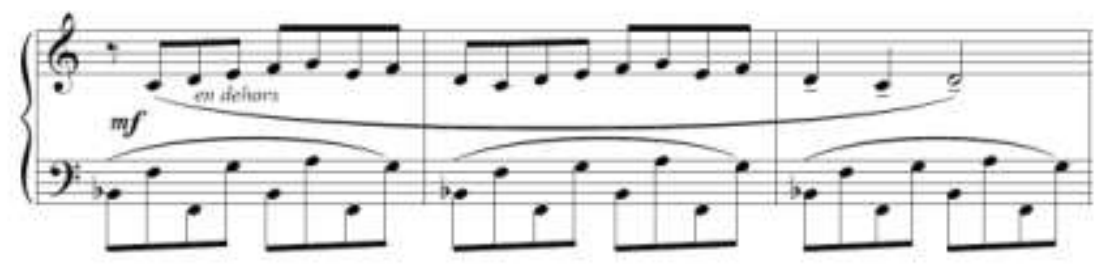

Quant à c, il s'agit d'une brève cellule dans laquelle la nomenclature rythmique contraste avec les croches régulières utilisées jusqu'ici.

Figure 4 : Francis Poulenc, Mouvements perpétue/s (I), mes. 12-13

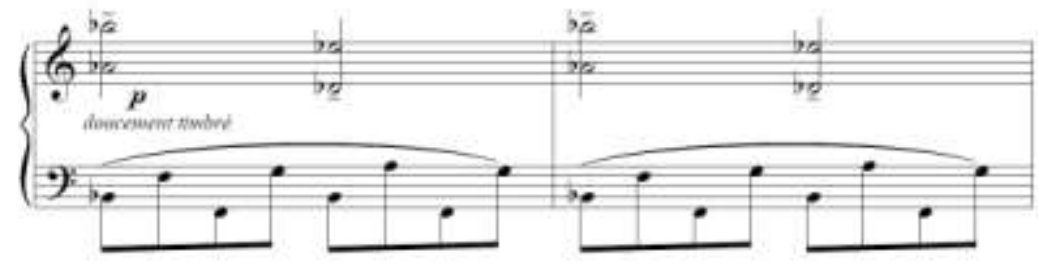

Les deux accords (deux neuvièmes vides en blanches) sonnent comme des cloches («doucement timbré » dit Poulenc) faisant tinter un mouvement binaire $\hat{5}-\hat{1}$ en MIb (sous-dominante de $S / b$ ). La notion de balancement est donc conservée ici, tout comme sur les notes-pivots constituant le motif $\mathbf{d}: \quad \hat{4}-\hat{1}-\hat{5}-\hat{1} \quad$ (si on reste en Mlb pour la main droite).

Figure 5 : Francis Poulenc, Mouvements perpétuels (I), mes. 14-15

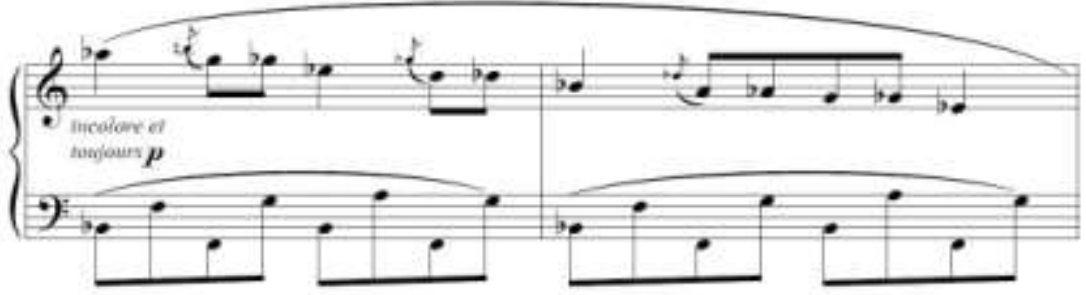


En outre, celui-ci introduit deux nouveaux éléments: une ornementation supérieure mordante à la tierce ou quarte supérieure et un glissement descendant de demi-ton. La cadence finale, indiquée «Très lent » et intervenant après un point d'arrêt propose davantage une mise en condition pour le numéro suivant qu'une prolongation de tonique que pourrait suggérer la pédale tenue.

Figure 6 : Francis Poulenc, Mouvements perpétue/s (I), mes. 22-24

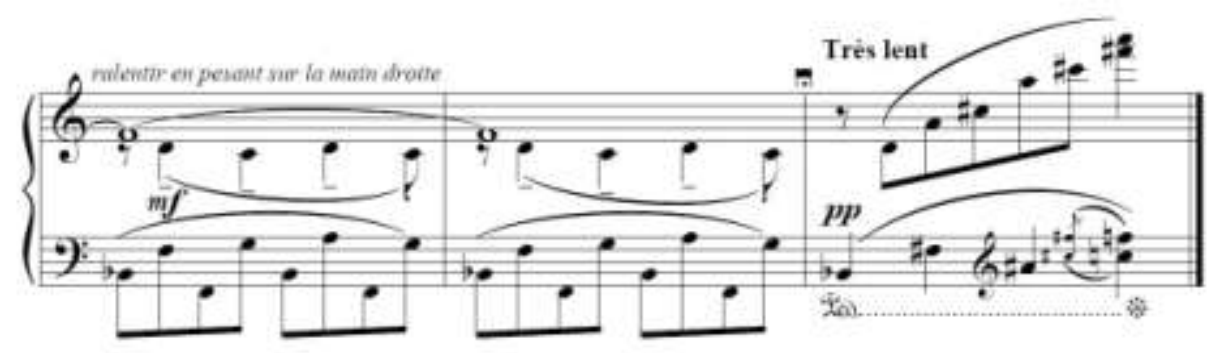

En effet, passé le premier temps sur S/b le déploiement des croches suivantes nous invite à entendre la tonalité de $R E$ (avec septième majeure) et le dernier temps est à nouveau consacré à un glissement au demi-ton inférieur sur une quarte à vide posée sur do (la dominante de dominante). Dans les mesures qui précédaient cette cadence, l'exploitation de la broderie finale de $\mathbf{a}$ et de $\mathbf{b}$ (ré-do-ré) nous avait préparés à une fin sur ré, mais avec tierce mineure. L'arrivée de la tierce majeure est un éclairage qui transforme le noir en blanc. Par ailleurs la superposition de ce fa\# de main droite sur le fa bécarre de main gauche est étonnant et réalise un savoureux mélange de blanc et de noir en décalage. L'ostinato de main gauche aura assuré une toile de fond uniforme.

La superposition d'antagonismes (au moins mélodiques) se poursuit dans le second moto perpetuo ${ }^{10}$. D'emblée, le premier motif se

10 Dans le manuscrit, il existe deux versions de ce deuxième Mouvement. Par ailleurs, l'œuvre pour piano a été révisée par Poulenc en 1939, puis en 1962. 
présente à deux voix en mouvements contraires. Cette fois la ligne conjointe ascendante affecte la main droite (sur le premier pentacorde de ré) et la ligne descendante à partir de ré est réservée à la main gauche.

Figure 7 : Francis Poulenc, Mouvements perpétue/s (II), mes. 1-2

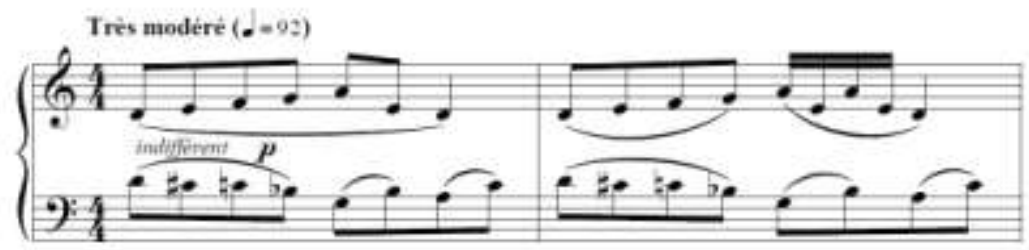

Pas de voix intermédiaire comme dans le morceau précédent, en revanche on note une forte présence de la quarte mélodique et de son renversement, la quinte, les deux couleurs intervalliques principales. Un antagonisme mélodique est également à relever mesure 7 lorsque la main gauche casse le mouvement conjoint alors prédominant pour travailler une ligne brisée en sixtes descendantes (sib-ré-mi-sol) distendant la cellule initiale (ré-mi-fa-so).

Figure 8 : Francis Poulenc, Mouvements perpétuels (II), mes. 7-8

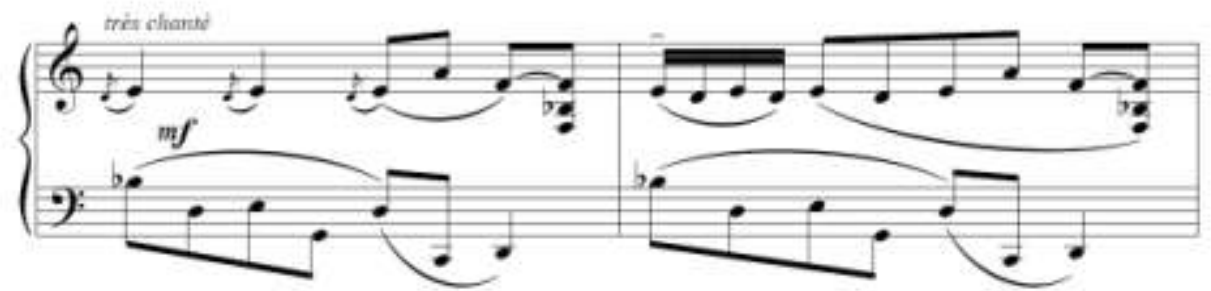

Par ailleurs, mais toujours sur un plan horizontal, un balancement $\hat{n} \wedge$ en octaves, reporté sur plusieurs registres et tinté par une petite note en anticipation apparaît dans l'ultime avatar du motif initial. 
Figure 9 : Francis Poulenc, Mouvements perpétue/s (II), mes. 11-12

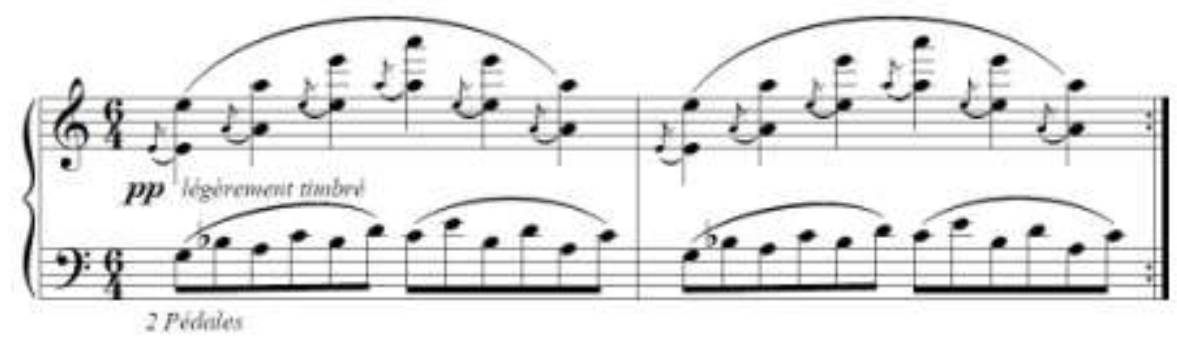

II est accompagné de tierces coulées en main gauche"1, contribuant à nimber de résonances harmoniques un large espace, geste qui se retrouve - mais différemment - dans la dernière mesure où un large glissando joignant le rés au rés se superpose à un mouvement cadentiel en $d o$.

Figure 10 : Francis Poulenc, Mouvements perpétue/s (II), mes. 13-14

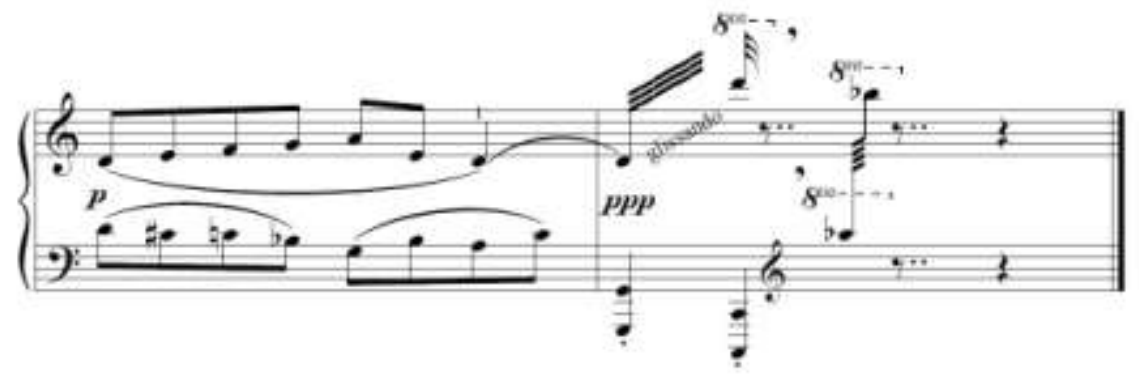

Voilà encore une conclusion en décalage, comme dans le morceau précédent, do étant la dominante du morceau suivant. Autre anticipation: une trille sur deux notes qui se transformera plus loin en trilles plus larges sur trois notes. Ici, elle a l'effet d'une couleur complémentaire jurant sur le glissando de ré et valorisant a contrario la cadence en do. Il s'agit d'un réel équilibre des couleurs « harmoniques » visant à valoriser la zone médiane de mélange, celle que nous avons vue si prégnante dans le tableau synoptique supra. L'approche colorée qui caractérise la composition musicale chez Poulenc se lit mieux encore

11 La tierce est le renversement de la sixte dont nous avons relevé la présence pour le motif a4, mesures 7-10. 
dans le domaine de la mélodie pour lequel Marjorie Running Warton déclare: "Poulenc similarly remarked that he rarely wrote descriptive accompaniments or word painting, but sought rather to invoke the colour of the poem ${ }^{12}$.

Ces quelques remarques confirment une appréhension essentiellement chromatique de l'œuvre musicale (au sens iconographique) dans laquelle Poulenc s'est fait peintre, attentif à la sonorité, comme le fut avant lui Claude Debussy. Notre titre « en blanc et noir » appliqué à cette œuvre exprime tant un jeu de contraste qu'une référence à Debussy, l'auteur d'une suite pour deux pianos dans laquelle ce titre marquait l'engagement politique de son auteur en 1915. D'abord baptisés Caprices, les trois mouvements qui constituaient cette pièce étaient indépendants et devaient être des expressions musicales du retour à la musique française «Grand siècle». Trois ans plus tard, le jeune Poulenc apporte sa pierre à ce qui aura pour nom «Néoclassicisme ». Lui, le futur «Six »13, l'admirateur de Stravinsky, contribue, par ses Mouvements perpétuels, à faire coïncider une esthétique nouvelle de la simplicité, de l'économie des moyens sur une toile de fond toute faite de déférence à l'égard de ceux qui l'ont précédé, nous allons le voir plus avant.

Nous venions de souligner le rôle de plaque tournante dévolu à l'extrême dernière mesure de cadence ${ }^{14}$. Cela nous rappelle le vœu émis

\footnotetext{
12 C'est l'auteur qui souligne. «Poulenc remarquait de même qu'il composait rarement des accompagnements descriptifs ou des peintures sonores du mot, mais il cherchait plutôt à évoquer la couleur du poème ». (C'est nous qui traduisons. Marjorie Running Warton, «Poulenc and Dufy », dans Sidney Buckland et Myriam Chimènes (dir.), Francis Poulenc, Music, Art and Literature, Aldershot, Ashgate, 1999, p. 189).

13 Lorsqu'on découvrit ces Mouvements perpétuels, ils furent donnés avec une création des Images à Crusoé de Durey et des œuvres de Milhaud, Auric, Tailleferre, faisant de cette audition, le premier concert des «Six 》 (cf. Myriam Chimènes (éd.), op. cit., p. 84 et p. 88 où dans une lettre à Valentine Gross - le 14 mars 1919 - le compositeur confirme que sera jouée, au même concert que les Mouvements, sa Sonate pour deux clarinettes).

14 II n'y a évidemment aucune autre cadence par ailleurs. L'évitement est total, même si les basses ne cessent de dessiner - de façon plus ou moins masquée les cernes de la chute 5-1.
} 
par Poulenc sur son manuscrit : «Ces trois pièces doivent être jouées et enchaînées l'une à l'autre et promptement enchaînées. Le tout doit se dérouler uniformément et d'une façon tout à fait incolore. Le pianiste doit oublier qu'il est virtuose ${ }^{\prime 15}$. Sa propre notification insiste donc sur la recherche sonore de neutralité équilibrée dans l'agencement mélodico-harmonique du tout. Des années plus tard, il considère avec recul les interprétations que les pianistes ont livrées à la postérité et il conclut :

Les grandes erreurs techniques qui défigurent ma musique de piano, au point de la rendre méconnaissable, sont: le rubato, l'avarice de pédale et le trop d'articulation de certains dessins en batteries ou arpèges qu'il faut, au contraire jouer très estompés16.

Il est donc de prime importance de tenir compte des nombreuses indications émaillant les Trois Mouvements perpétuels, en adaptant peut-être la recommandation sur les batteries ou arpèges, aux figures d'ostinati qui traversent les trois pièces.

Avec le troisième moto perpetuo, des éléments déjà entrevus se confirment. En effet, la tonalité de $F A$ annoncée par sa dominante dans la dernière mesure du morceau précédent est de suite affirmée. La première cellule, anacrousique, propose un trajet $\hat{5}-\hat{1}$ en quatre notes conjointes ascendantes dessinant l'intervalle de quarte déjà tant exploité précédemment.

\footnotetext{
15 Cité par Carl B. Schmidt, The Music of Francis Poulenc (1899-1963). A Catalogue, Oxford, Clarendon Press, 1995, p. 31. Nous n'avons pas eu la possibilité de vérifier le manuscrit détenu aux Etats-Unis, mais deux coquilles se sont insérées dans la citation de Schmidt que nous avons du mal à imputer à Poulenc; le cas échéant, il aurait fallu les signaler par un sic explicite.

16 Francis Poulenc, Entretiens, op. cit., p. 32. C'est nous qui soulignons.
} 
Figure 11 : Francis Poulenc, Mouvements perpétue/s (III), mes. 1-3

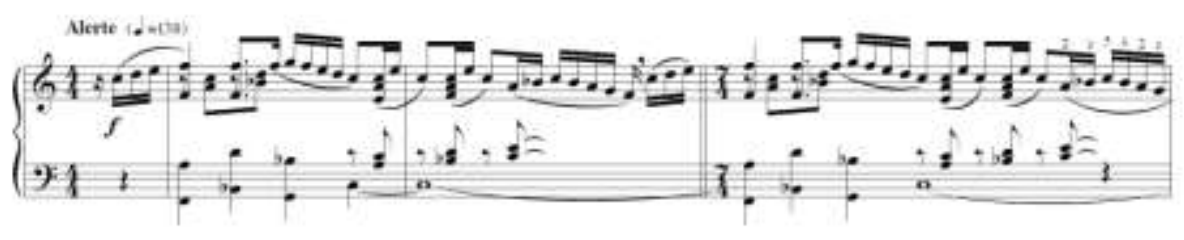

Elle est immédiatement suivie d'un versant descendant de ces quatre notes conjointes. Quant à la main gauche, elle glorifie encore le mouvement cadentiel (la chute de quinte), non sans avoir, auparavant, enchaîné des accords de dixièmes qui rappellent les neuvièmes de la mesure 12 dans le premier morceau. Le saut de quarte est encore le pivot du troisième motif en basse (ré-sol) annonçant une page posée sur so/mineur à laquelle Poulenc superpose une main droite plutôt insérée dans un moule de ré mineur17. II ne faut cependant pas voir là une intention polytonale, juste des cernes noirs autour de taches de couleur. Remarquons par ailleurs que la tonalité de ré est à distance de tierce de $F A$, autre intervalle que nous avons déjà constaté être très présent ${ }^{18}$. La main droite oscille sans cesse entre des triades de ré et de $F A$ créant une instabilité qu'accentue la main gauche dont les harmonies évoquent sol mineur ou majeur - par la versatilité du si, indifféremment bécarrisé ou bémolisé - et qui devient soudainement, l'espace de deux mesures, la sixte ajoutée d'un accord de ré19.

17 À l'exclusion des mesures 20 à 23 où la basse est un arpège brisé de $f a 7 e$. Signalons également le trouble localisé que constitue l'alternance des accords posés sur la (mes. $40 s q q$ ) à l'état fondamental puis en sixte et quarte, soit un enchaînement la-ré (à distance de quarte encore).

18 Les parties intérieures du premier et troisième motif sont également en superpositions de tierces. On les retrouve commandant les mouvements de basse des mesures 12-13.

19 Voir les mesures 28 à 36. 
Figure 12 : Francis Poulenc, Mouvements perpétuels (III), mes. 28-36

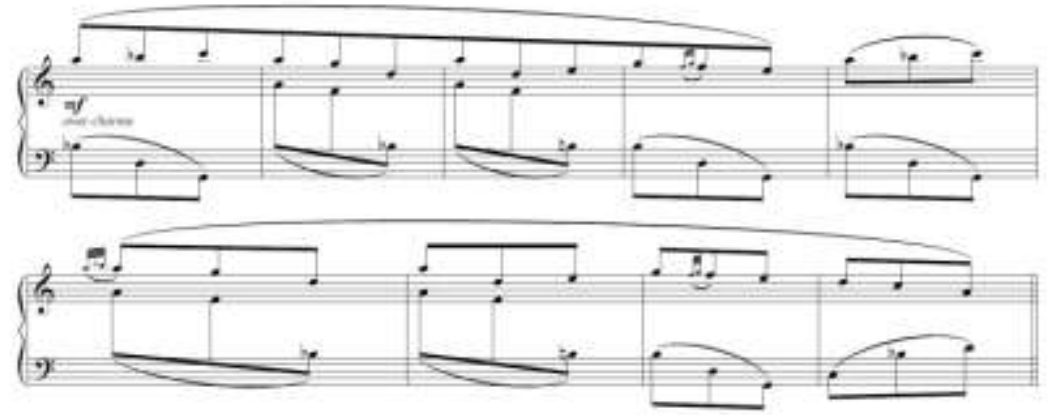

Cette grande mouvance harmonique contribue à flouter les contours tonals et crée un effet de trouble permanent. Autre vibration de ce troisième mouvement, la trille des mesures 45-46, puis 53-54 qui s'ajoute aux mordants dont nous avions déjà signalé la présence précédemment, se chargeant d'orner quelques notes-clés tout en évoquant un siècle passé vers lequel maints compositeurs français se tournent alors.

Figure 13 : Francis Poulenc, Mouvements perpétuels (III), mes. 45-46+ $53-54$
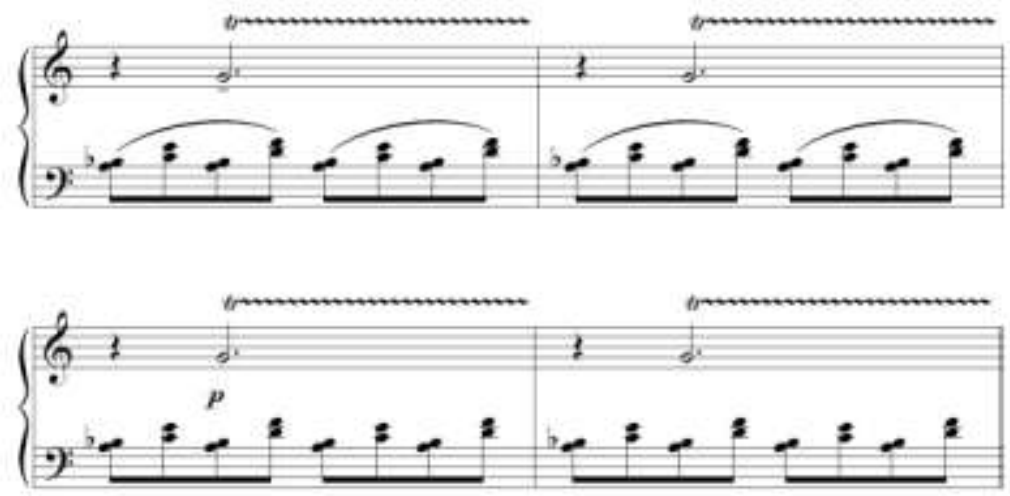

L'on retrouve un procédé déjà utilisé dans le premier moto perpetuo, à savoir une main gauche conservée à l'identique sous une 
main droite au motif transposé, ici un ton en dessous (mesure 51 , sol sol sol fa mi ré do) ${ }^{20}$, entraînant ipso facto un nouvel effet de superposition de couleurs (ici $s o /$ et $D O$ ) imposant cette dernière tonalité (dominante de $F A$ ) quelques mesures avant la cadence finale.

D'ailleurs tandis que la dernière mesure fait entendre une conclusion en $F A$ (linéairement $f a-s o l-l a$ ) en partie supérieure, la main gauche conclut en $D O$ (ré-sol-do) avant qu'un accord final superpose un accord de la à ce que nous souhaitons voir comme un accord de ré dont la tierce et la quinte ont été respectivement remplacées par une seconde et une sixte, ultime geste de détournement ${ }^{21}$.

Figure 14 : Francis Poulenc, Mouvements perpétuels (III), mes. 51-57
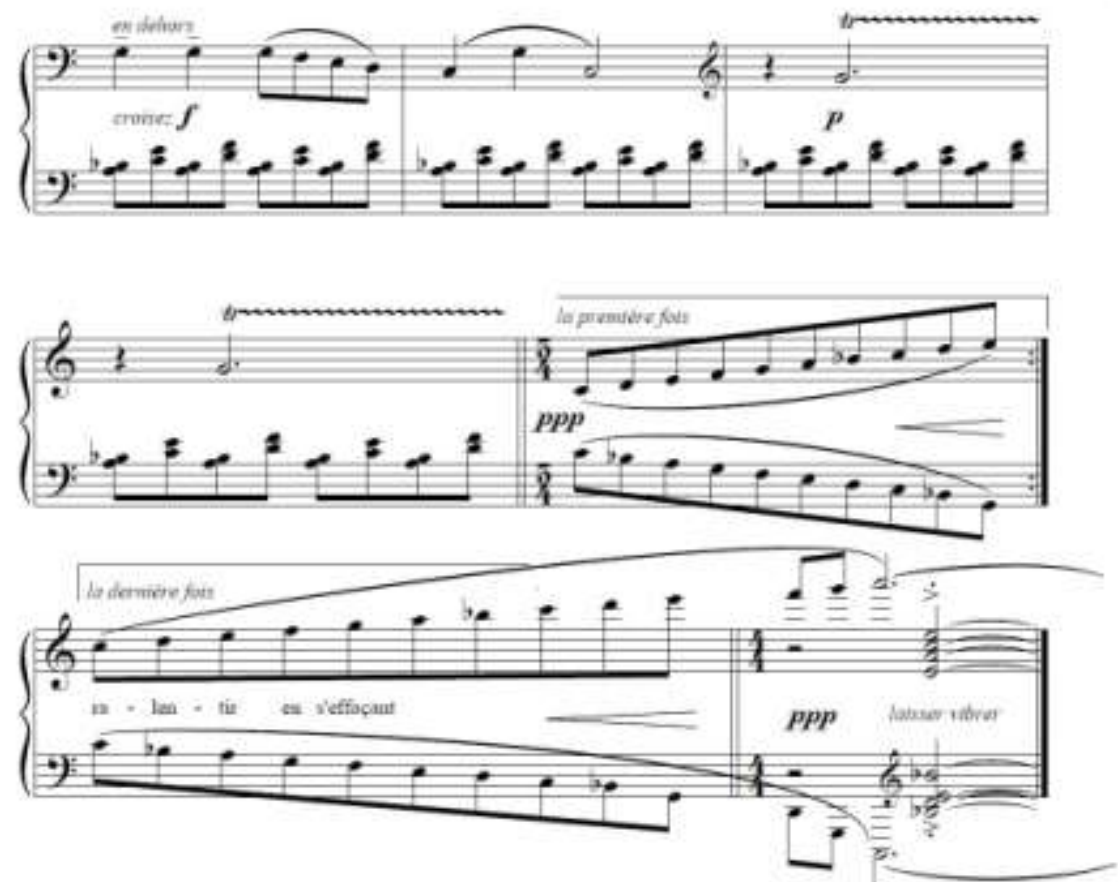

20 Cf. la transposition à la tierce dans le premier mouvement, mesure 10 .

21 Il va sans dire qu'il est loisible de l'interpréter tout autrement, en S/b par exemple. 
La permanence de certains éléments et les enchaînements d'un mouvement à l'autre nous poussent à conclure à une pensée fondatrice de nature cyclique. Par ailleurs, les évitements et décalages que l'on vient de mettre en évidence dans l'écriture harmonico-mélodique sont également visibles d'un point de vue structurel, nous allons en parler. Or, l'approche colorée que nous avons essayé d'imposer est corroborée par le compositeur lui-même qui entreprit à deux reprises d'orchestrer (en 1925) et d'instrumenter (en 1946) son œuvre22. II semblerait donc que ces Trois mouvements perpétuels s'y prêtent bien. D'ailleurs, après leur audition aux concerts Straram du 7 avril 1927, un critique pouvait remarquer: "Francis Poulenc a récemment orchestré ses Mouvements perpétuels. II y a apporté des soins attentifs, la franchise d'une peinture sans mélange, une netteté incisive ${ }^{23}$ ». Jascha Heifetz en fera également une version pour piano et violon en 1930, tandis que Poulenc lui-même tentera celle à deux pianos $^{24}$ et que d'autres encore se lanceront dans diverses adaptations de l'œuvre. Nul doute que ces différentes versions contribuèrent au succès de l'œuvre.

\section{La structure interne}

La référence générique est incontestablement Satie. Pourtant, si l'ostinato de basse est indéniable dans les deux premiers Mouvements (en dépit de changements affectant les dernières mesures du second), le troisième adopte une structure faite de juxtapositions et de retours qui masque quelque peu le geste obstiné. Les Vexations du Maître d'Arcueil sont cependant un modèle pour un compositeur qui s'en était rapproché. Ne lui avait-il pas envoyé ses affectueuses pensées au moment du décès

\footnotetext{
22 L'arrangement pour neuf instruments est conçu pour flûte, hautbois, clarinette en Sib, basson, cor, violon, alto, violoncelle et contrebasse.

23 André Georges, Les Nouvelles littéraires, 30 avril 1927, p. 9.

24 Probablement avant 1951, mais cette version n'existe plus que sur un enregistrement et le manuscrit est perdu.
} 
de son père ${ }^{25}$ ? Les années 1913-1914 sont d'ailleurs celles où Satie introduit de plus en plus de para-textes dans ses partitions. Sans en adopter l'humour ou la causticité, Poulenc est toutefois fort prolixe en la matière avec ces Mouvements perpétuels (nous avons relevé une vingtaine d'indications littéraires pour un total de 95 mesures). Après les Vexations, Satie conçoit, en 1903, ses Morceaux en forme de poire (pour quatre mains) qui apparaissent comme une réponse au reproche de Debussy qui ne voyait aucune forme dans ses pièces. Une décennie après, les Descriptions automatiques (1913) sont la réponse « impressionniste » de Satie à l'académisme de boursouflure, trois pièces agrémentées de réflexions cocasses se prolongeant peu après dans les trois morceaux formant les Embryons desséchés de 1913. Nos trois brefs morceaux de Poulenc s'inscrivent donc dans cette lignée de brèves trilogies, au profil simple, un rien lancinant, et surtout au charme poétique fait de retenue et de non-conformisme. Poulenc n'ira pas jusqu'à donner l'indication satiste figurant en tête des Vexations: "Pour se jouer 840 fois de suite ce motif, il sera bon de se préparer au préalable, et dans le plus grand silence, par des immobilités sérieuses ». Cependant, une barre de reprise est prévue quelques mesures avant la fin, pour chacun des Mouvements. Et rien n'indique qu'une seule redite est prévue, puisqu'au dernier, une terminaison de "première fois » est envisagée, puis celle de "la dernière fois ». Ce qui est sûr, c'est que Poulenc n'envisage pas d'omettre les reprises. Pour preuve, sa colère lorsqu'il découvre que Kling a fait perforer ses Mouvements pour pianola, sans son consentement. II dit à Milhaud être fort mécontent car les reprises ont été supprimées et le premier mouvement «est mal mécanisé, le second aussi (manque de pédale, mouvements faux, nuances exagérées), mais le dernier est absolument ravissant, beaucoup mieux qu'au piano surtout dans le passage en dixièmes pour la main

25 Voir la lettre de Satie à Poulenc, le 16 juillet 1917 (Monsieur Émile Poulenc est décédé le 15 juillet 1917), dans Myriam Chimènes (éd.), op. cit., p. 53. Francis Poulenc a rencontré Satie en 1916, par l'intermédiaire de son professeur Ricardo Viñes. 
gauche $26 »$. Ce propos est intéressant car il précise la vision de Poulenc sur son second mouvement qu'il voulait vraiment avec pédale (les deux sont indiquées deux mesures avant la reprise) et avec une expression de retenue, de demi-teinte que nous avons déjà soulignée. D'ailleurs, sur son manuscrit, l'on peut lire la recommandation suivante : "Le no [ill.] se jouera trois fois de suite : la première sur un ton indifférent. La seconde très rubato, la troisième avec rage en donnant une accentuation sur chaque note 27 ». Nous émettons l'hypothèse qu'il s'agit du second Mouvement. Quoi qu'il en soit, il est clair, une fois de plus, que la reprise est obligatoire et qu'elle excède la redite unique. À Milhaud, Poulenc révélait également son écoute du troisième Mouvement dont la partie $\mathbf{A}$ qui sonnait mieux au pianola qu'au piano devait donc être assez «mécanique» de conception; c'est sans doute la pièce la plus «populaire» dans l'esprit. Sa structure même suppose un pseudorefrain (voir le schéma en annexe 1).

Le dernier Mouvement est un bon exemple des principes architecturaux de Poulenc. En effet, la répétition des motifs à la base du moto perpetuo s'effectue en employant maint procédés de variations. Aussi le motif $\mathbf{a}_{1}$ est-il de suite répété dans un changement de métrique, lui ôtant peut-être son accent de cinquième temps, lequel correspondait à une réitération dactylique interne. De même qu'a $a_{3}$ est lui-même constitué d'une mesure en deux moitiés égales (ou sensiblement), ce qui revient à énoncer quatre mêmes cellules (la première étant à l'octave inférieure se détache un peu).

26 Lettre de Poulenc à Milhaud [début août 1924], dans Myriam Chimènes (éd.), op. cit., p. 232.

27 Nous n'avons pas eu accès au manuscrit. Cité par Carl B. Schmidt, op. cit., p. 31 qui précise que cette remarque se situe en p. 17 du manuscrit. 
Figure 15 : Francis Poulenc, Mouvements perpétuels (III), mes. 6-7

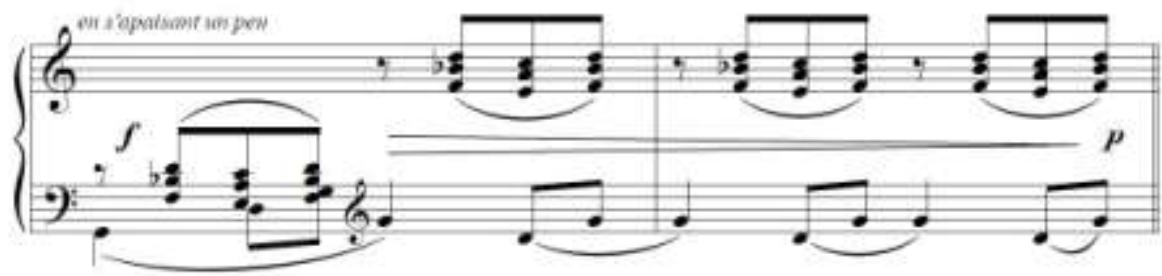

Nous avions déjà signalé le changement de couleur harmonique en partie B qui fait montre d'un nouveau procédé que nous choisissons de nommer « greffe ».

Figure 16 : Francis Poulenc, Mouvements perpétue/s (III), mes. 8-19
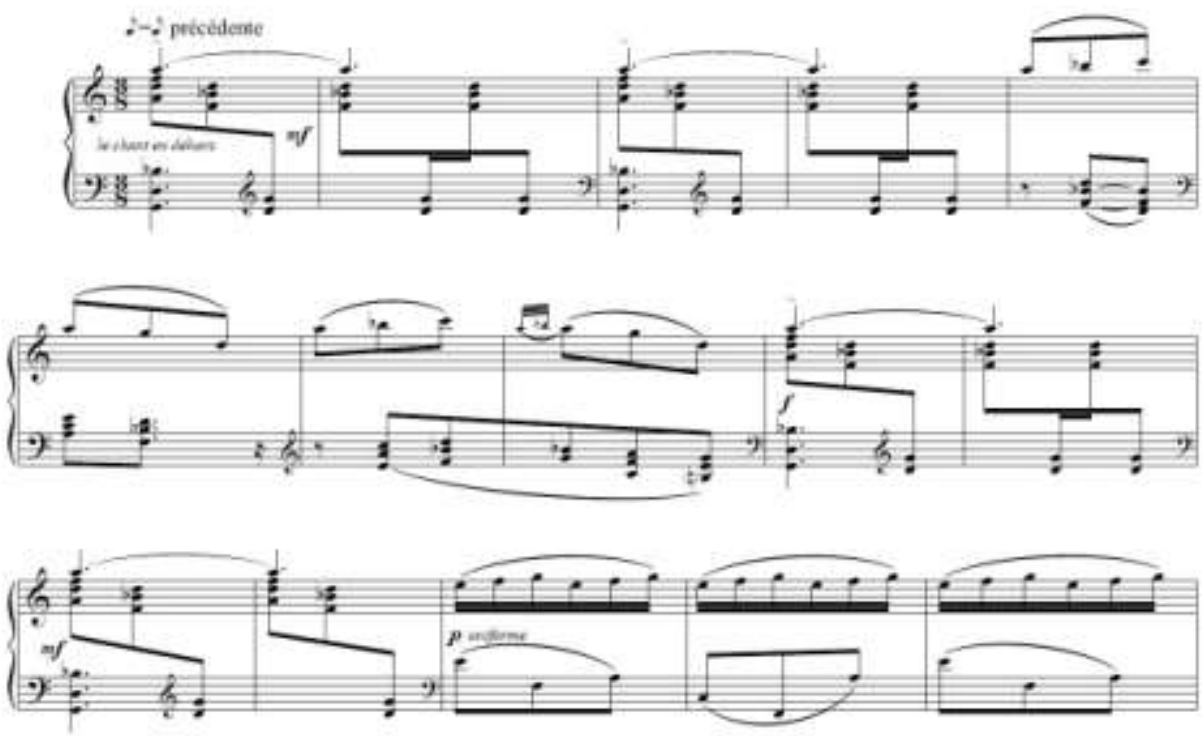

En effet, $b_{1}$ (2 mesures) est immédiatement réitéré - remarquons encore qu'il est lui-même son propre monnayage en seconde mesure bientôt interrompu par une greffe de $b_{2}$, également en $2 \times 2$ mesures, avant le retour immédiat de $\mathbf{b}_{1}$. Pourtant, rien n'est jamais tout à fait 
exact, les terminaisons varient ${ }^{28}$. Nouvelle greffe : la trille développée sur trois notes de $\mathbf{b}_{3}$, donnant enfin un mouvement tournant à l'ensemble.

Figure 17 : Francis Poulenc, Mouvements perpétue/s (III), mes. 20-23

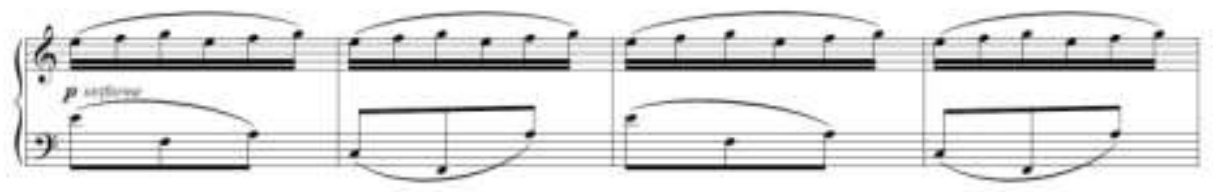

Après un ultime retour de $\boldsymbol{b}_{1}$, le motif $\mathbf{b}_{\mathbf{4}}$ (issu de $\mathbf{b} 2$ ) se redit avec une terminaison différente la seconde fois.

Figure 18 : Francis Poulenc, Mouvements perpétue/s (III), mes. 28-35

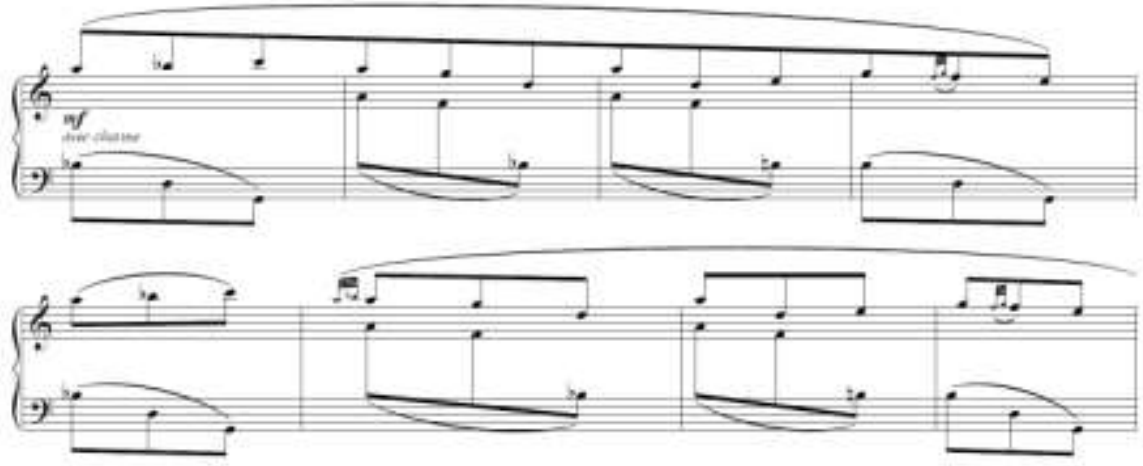

La dernière partie de ce Mouvement emploie encore le procédé de greffe par la trille de $c_{2}$ - à l'identique dans la partie C' - ainsi que le procédé d'amplification avec c'1 qui instaure deux voix en octaves et une main gauche partagée entre les deux mains. La dernière exploitation de $c_{1}$ s'effectue sur une main gauche identique, mais avec un motif mélodique de main droite démarrant un ton au-dessous (déjà dit supra), respectant la tri-répétition de cette note de tête, mais se poursuivant par quatre croches conjointes issues des quatre doubles du motif originel. La

28 La main gauche des mesures 14-15 n'est pas celle des mesures 12-13 ; la mesure 19 n'est pas la réplique exacte de la mesure 17. 
seconde mesure est occupée par un mouvement cadentiel (do-sol-do) qui rappelle la cadence du Mouvement précédent (cf. supra), et conférant de ce fait, une couleur de $D O$ à ce qu'on entendait jusque là dans un camaïeu de ré. En effet, la partie $C$ était centrée sur un la autour duquel tout tournoyait. L'usage d'une note de référence, d'une couleur dominante cernée de ses dégradés est également à signaler dans le deuxième Mouvement. Dans celui-ci, c'est la note mi qui joue un rôle centrifuge intéressant tout au long de la pièce (voir la structure en annexe 2). Elle est omniprésente, parfois très ostensiblement se répétant en main droite, en acciacature (mes. 5-7-9-10), parfois plus discrète contenue dans les accords.

Figure 19 : Francis Poulenc, Mouvements perpétue/s (III), mes. 40-50

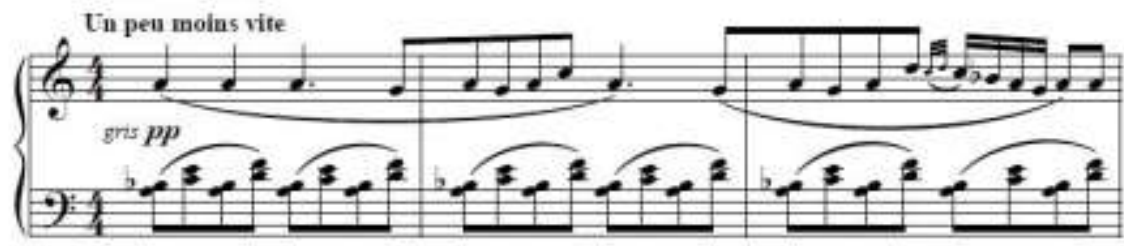

les derex poldales
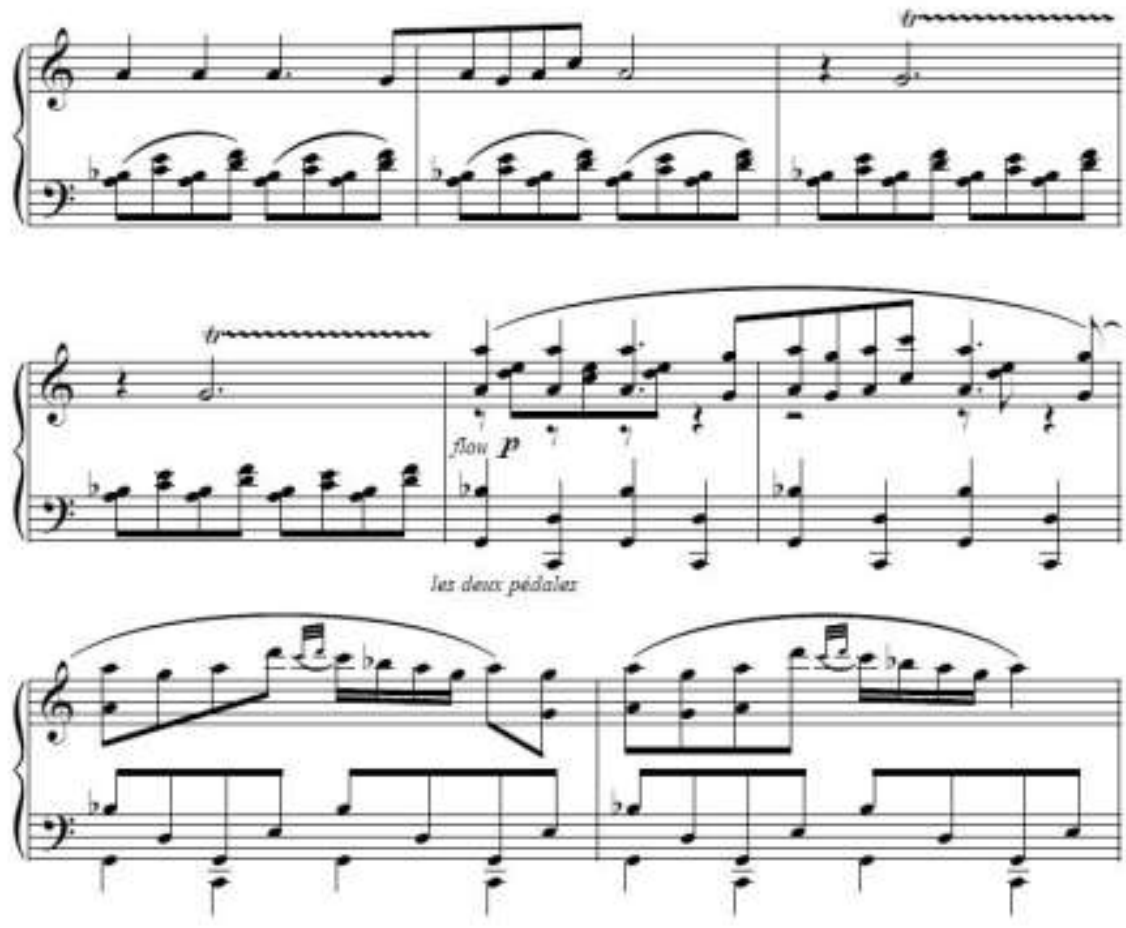
D'une façon générale l'examen du schéma formel confirme le procédé de construction employé par Poulenc qui additionne des cellules, en les variant insensiblement (notamment via le monnayage interne, la broderie, le report de registre) mais qui évite tout développement thématique, tournant le dos à une tradition germanique par trop envahissante.

Privilégiant le travail sur la résonance dont nous avons déjà parlé, ces concaténations motiviques qui virent aussi à la juxtaposition (cf. mes. 7-10) ne sont pas sans évoquer la figure de Ravel, admirée de Poulenc qui voulait être introduit auprès du maître de L'Heure espagnole par l'intermédiaire de Viñes. Ce qui fut fait ${ }^{29}$. Aussi n'est-il pas étonnant de lire dans ces premières compositions de Poulenc une référence structurelle à l'un des compositeurs appartenant à son panthéon. Lucie Kayas a d'ailleurs récemment souligné cette filiation affirmant : "Poulenc also refers to Ravel on questions on form, taking his advice to use as a model for new works the formal plan of an acknowledged masterpiece in the repertoire, as in the case of his Trio for piano, oboe and basson ${ }^{30} »$.

Pour notre part, nous estimons que la Sonatine de Ravel composée en 1905 pour ses amis Ida et Cipa Godebski peut avoir joué ce rôle de matrice structurelle et motivique. Par exemple, la prolongation de la tenue du motif $b_{1}$ puis $b_{2}$ dans le dernier Mouvement de Poulenc adopte un profil mélodique que nous trouvons peu éloigné des mesures 6-10 du premier mouvement de la Sonatine (Fig.20).

Évidemment, l'on constate le travail de monnayage immédiat entrepris par Ravel, à l'égal de ce que fera Poulenc. Un peu plus loin, la stagnation sur la cellule de broderie (Fig. 21 : si-do, mes. 13-15)

\footnotetext{
29 En effet, dans une lettre du [14 octobre 1917] Poulenc remercie Viñes de l'avoir recommandé à Ravel (voir Myriam Chimènes (éd.), op. cit., p. 57).

30 Lucie Kayas, «Francis Poulenc disc jokey », dans Sidney Buckland et Myriam Chimènes (dir.), op. cit., p. 374 : "Poulenc se réfère aussi à Ravel pour les questions de forme, adoptant son conseil d'utiliser comme modèle pour de nouvelles œuvres, le plan formel d'une œuvre majeure bien connue du répertoire, comme dans le cas de son Trio pour piano, hautbois et basson» (c'est nous qui traduisons).
} 
rappelle celle que nous avons soulignée dans le premier Mouvement (rédo). Après la barre de reprises, Ravel fait sonner des mi syncopés et décale lui aussi les temps forts par ses liaisons (Fig. 22 : mes. 29-34).

Figure 20 : Maurice Ravel, Sonatine (I), mes. 6-10

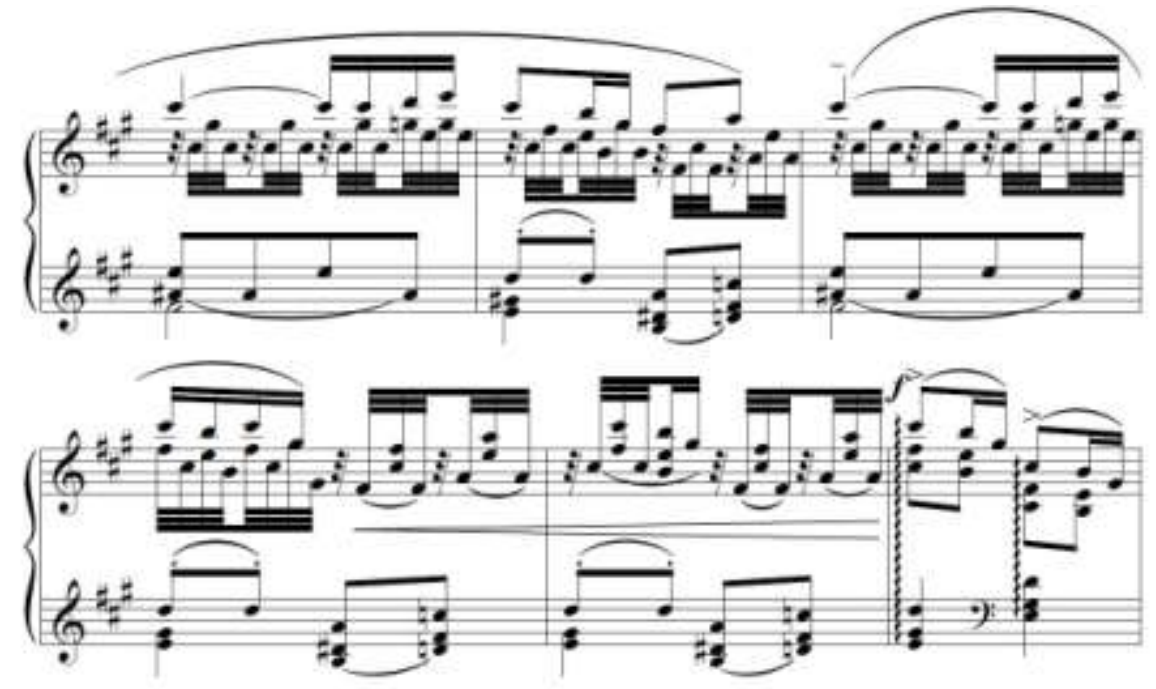

Figure 21 : Maurice Ravel, Sonatine (I), mes. 13-16

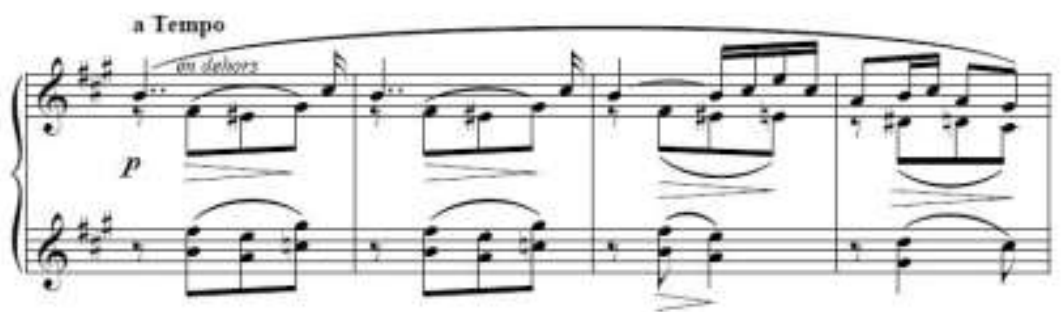

Figure 22 : Maurice Ravel, Sonatine (I), mes. 29-34

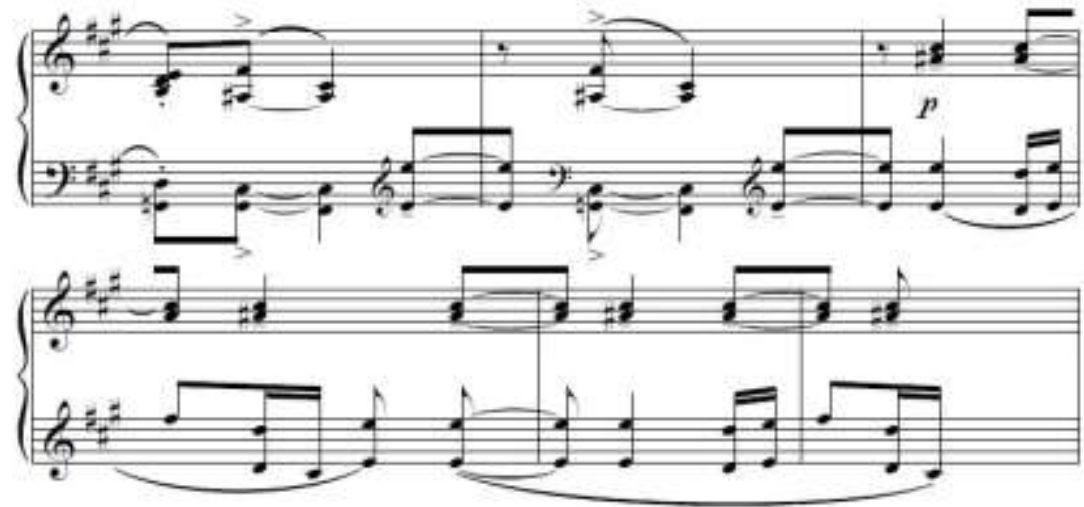


Remarquons également sa triade de ré\# en main gauche (mes. 37-40) qui présente - comme nous l'avons chez Poulenc - la triade en éclairage successivement majeur et mineur (III/31-34 chez Poulenc).

Figure 23 : Maurice Ravel, Sonatine (I), mes. 37-40

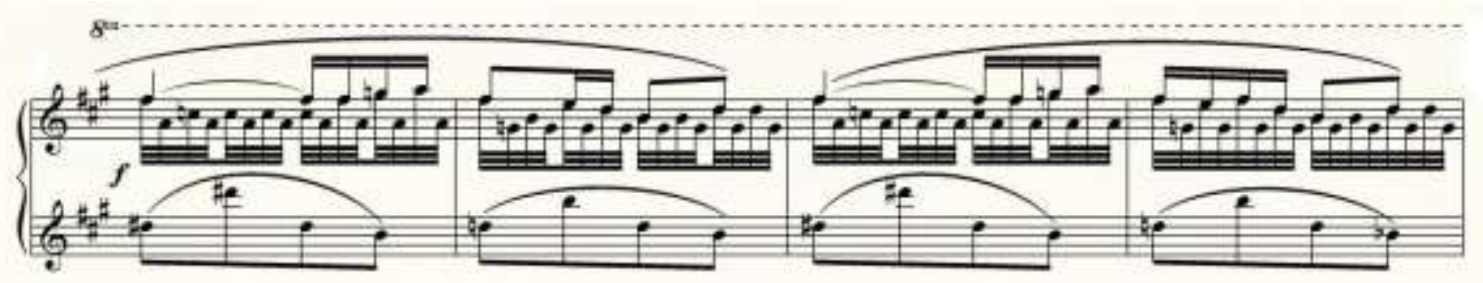

Quelques mesures plus loin, les secondes ravéliennes de main gauche (mes. 48-53) rappellent singulièrement ce que nous avons appelé les acciacatures de mi dans le second Mouvement (II/9-10).

Figure 24 : Maurice Ravel, Sonatine (I), mes. 48-53

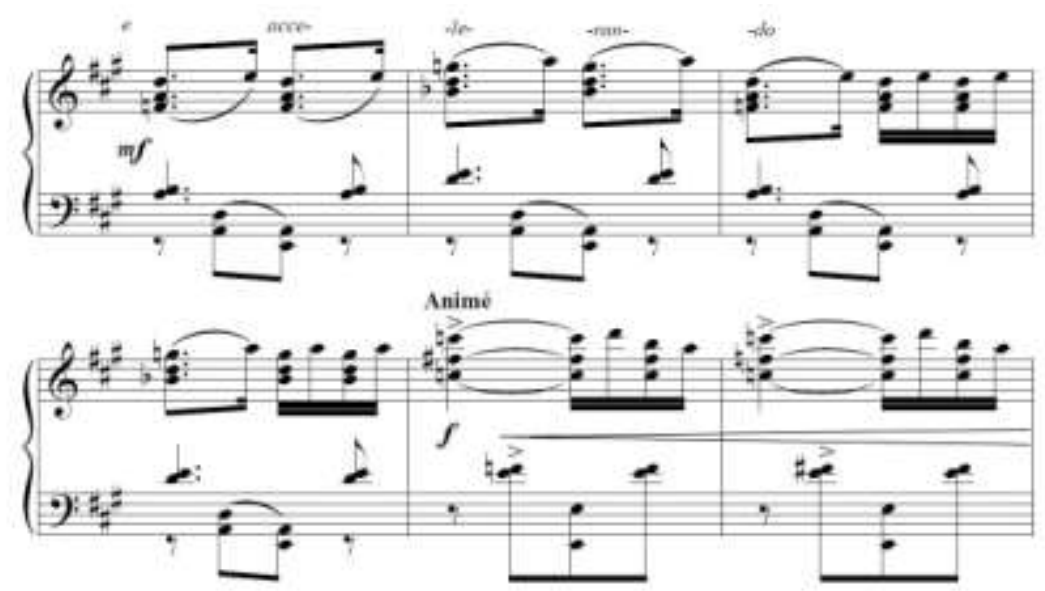

Si l'on parcourt rapidement maintenant le second mouvement de la Sonatine, on est frappé d'emblée par le dessin mélodique du thème initial : $1-5-1-5-1$ qui affirme les crêtes de $\mathbf{a}_{1}$ (ou $\mathbf{a}_{1}{ }_{1}$ ) chez Poulenc (II/1-2). 
Figure 25 : Maurice Ravel, Sonatine (II), mes. 1-2

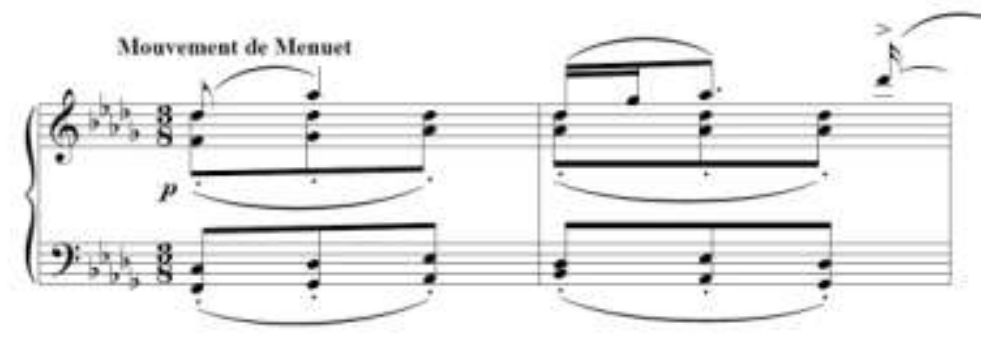

De suite après la première barre de reprise - qui intervient dès la mesure 12 - Ravel choisit d'orner par un mordant ou par des notes coulées descendantes de très brefs motifs en deux mesures avec répétitions internes qui rappellent divers moments déjà considérés chez Poulenc $^{31}$. Le troisième mouvement ravélien est une sorte de Toccata dont le matériau thématique semble issu de son premier mouvement (par exemple mes. 95-96). Comme ailleurs, les immobilisations sur des formules (cf. mes. 91-94) confinant à un moto perpetuo et les reports de cellules à l'octave sont des procédés amplement utilisés par Poulenc.

Figure 26 : Maurice Ravel, Sonatine (III), mes. 91-94

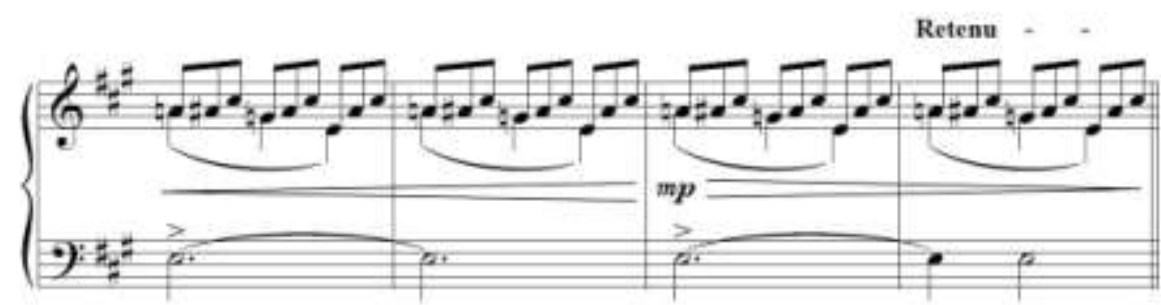

En outre, une étude intervallique un peu poussée nous autorise à considérer la quarte et la quinte comme des intervalles fondateurs dans la Sonatine et nous avons vu toute l'importance qu'ils revêtaient également chez Poulenc ${ }^{32}$. Dans le même ordre d'idée, le martèlement fa-sib (1-5) des mesures 147-151 (avec alternance des mesures à 5/4,

$31 \mathrm{I} / 10$ ou I/14-16; III/31-35 ou III/42, III/49-50.

32 Voir dans la Sonatine les sauts de quartes ou de quintes ascendantes (III/106107, puis 120-121). 
$4 / 4$, puis $3 / 4$ ) rappellent singulièrement les mouvements fonctionnels de basse soulignés chez Poulenc.

Figure 27: Maurice Ravel, Sonatine (III), mes. 147-151

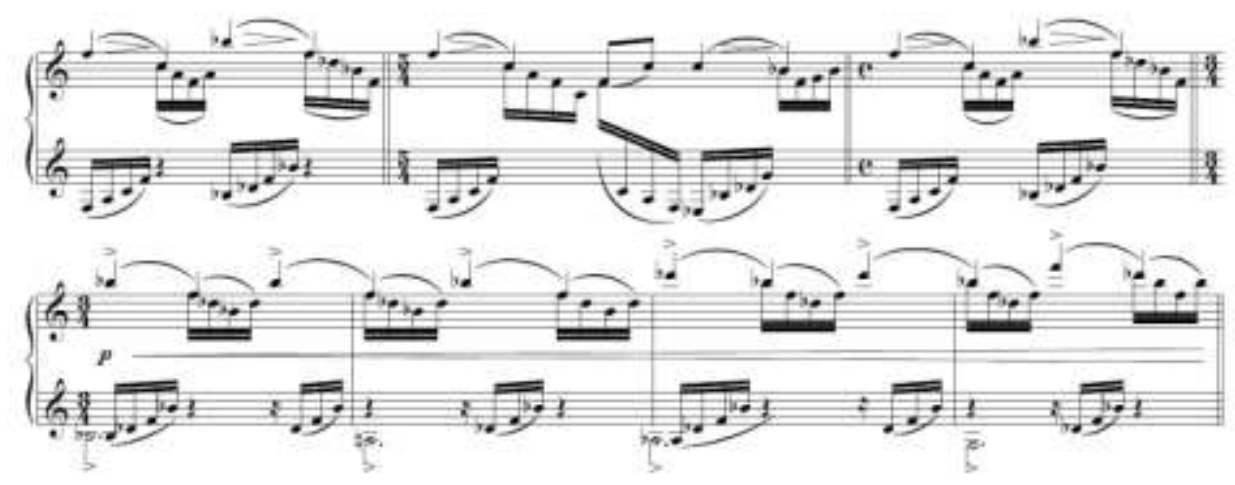

Quant aux ultimes cadences ravéliennes, elles possèdent déjà les éléments déstabilisateurs dont se délectera le compositeur de Noizay. Pour exemple, la fin du premier mouvement de la Sonatine s'achève en fa\# majeur avec un so\# $_{4}$ qui résonne en main droite et plusieurs des cadences opèrent par glissements, comme celle du premier des Mouvements de Poulenc.

Le premier motif de celui-ci (voir la structure en annexe 3) consacre un profil mélodique catabasique dans une écriture fluide à deux ou trois voix. Nous retrouvons les mêmes procédés déjà décrits, à savoir celui de la «greffe » (voir l'utilisation du motif $c$ ou $b_{1}$ ), la volonté subversive (par les décalages, de la cadence finale ou de la transposition de $a_{2}$ ). La permanence est alors exploitée dans la figure invariable de main gauche. Cette dernière se déroule sur une mesure et délimite par ses grands intervalles une ligne mélodique supérieure conjointe ( $\mathrm{fa}-\mathrm{sol}$ la-sol) sur un balancement $\hat{1}-\hat{5}$ (sib-fa) dont nous avons parlé au début. II est exclu de faire ressortir de quelque manière que ce soit l'enchevêtrement de ces deux lignes qui n'apparaît que comme résultante sonore inopinée. Le phrasé expressif indique d'ailleurs bien qu'il faut concevoir cet ostinato à la mesure et non de façon plus 
globale. Et ce, contrairement à l'ostinato qui se dessine au centre du "Mouvement» de Debussy, troisième pièce de ses Images (1 ère série, 1904-1905).

Figure 28: Claude Debussy, Images / (« Mouvement »), mes. 89-95

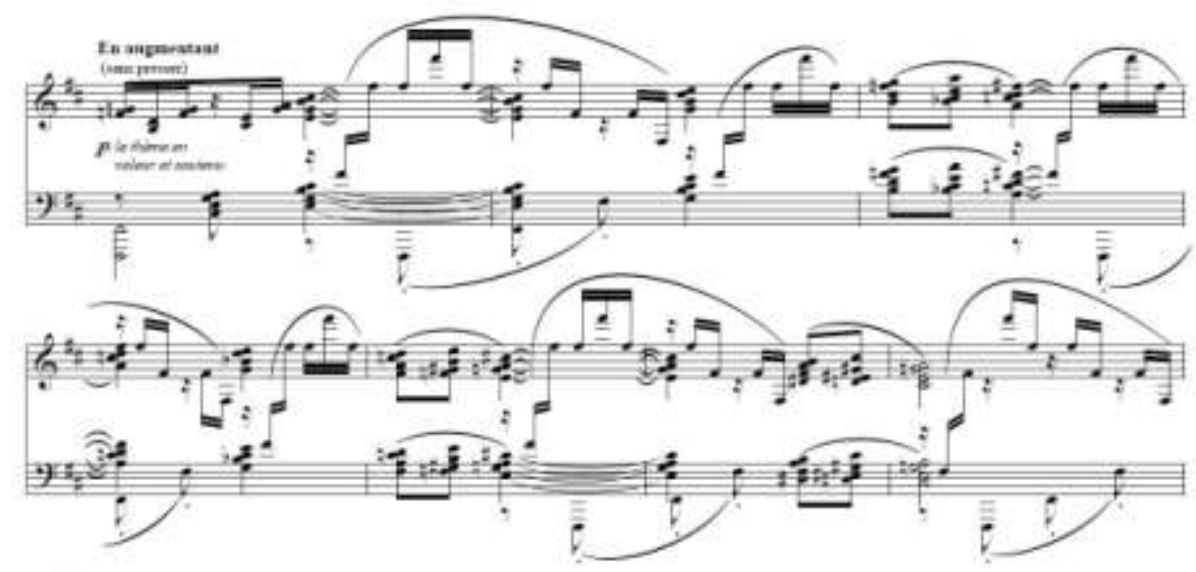

Nous avons cité Satie et Ravel, mais le compositeur qui a le plus compté dans la jeunesse de Poulenc, c'est assurément Debussy. Ce «Mouvement » debussyste est probablement à prendre en compte dans la galerie des modèles pour l'œuvre qui nous préoccupe ici. On y découvre des moto perpetuo en mouvements contraires agités de micro-variations internes; mais également le report d'une même note d'octave en octave, comme ce fa\# partagé entre la main gauche et la main droite ${ }^{33}$, dont le trajet est troué par des grappes d'accords de $2 \times 4$ sons (souvent des septièmes). Fasciné par Debussy, Poulenc n'eut de cesse d'en masquer l'emprise et il figure en tête des six compositeurs du xxe siècle qui l'ont le plus marqué : Debussy, Stravinsky, Satie, Falla, Ravel, Bartók et il ajoute Prokofiev34. Il reconnait que «l'on sent

33 Voir les mesures 89 à 114 des Images de Debussy (III - « Mouvement »). C'est Viñes qui les a créées le 6 février 1906.

34 En réponse à Rostand. Voir Francis Poulenc, Entretiens avec Claude Rostand, op. cit., p. 80. 
constamment la présence du grand Igor » dans sa musique, alors qu'il y "a peu de debussysme », mais il avoue de suite que "c'est Debussy, sans aucun doute, qui [l'] a éveillé à la musique », même s'il doit énormément à Satie «plus encore esthétiquement que musicalement ${ }^{35}$ ». L'on comprend cependant son agacement à la lecture de conférences musicales dues à diverses plumes dont celle de Maurice Boucher qui attaque violemment Debussy ou Ravel à mi-mots les nommant «les impressionnistes qui peignent en touches sonores et dont les œuvres sans émotion sont une sorte de dentelle transparente ${ }^{36} »$. Poulenc s'en offusque même s'il est entré dans une période de distanciation avec Debussy, saine distance sans doute qu'il se remémore trente-cinq ans plus tard en ces termes :

En dépit d'une crise anti-debussyste par self-défense à l'époque où j'ai connu Satie, en 1917, Debussy est toujours resté le musicien que je préfère après Mozart. Je ne peux pas me passer de sa musique. C'est mon oxygène. D'ailleurs la réaction des "Six » était dirigée contre le debussysme, et non contre Debussy. II faut toujours renier, pour un temps, à vingt ans, ce qu'on a idolâtré, de peur de se couvrir de lierre37.

Il est clair que ce qui est à bannir, ce sont les étiquettes dont on affuble les artistes de la première moitié du xxe siècle, à une époque où fleurit - comme jamais auparavant - une multitude de courants esthétiques, où s'entrechoquent des tendances contradictoires, où l'interchangeabilité des arts se lit dans le domaine terminologique, enfin, où règne en maître l'éclectisme le plus éclaté et ce, particulièrement

35 Les quatre expressions sont extraites de Francis Poulenc, Entretiens, op. cit., p. 180-181. Mais ailleurs, il indique que ses « seuls maîtres sont Bach, Mozart, Satie et Stravinsky » et Debussy a disparu. Cf. lettre de Poulenc à Otto Marius Kling le 6 septembre 1919, dans Myriam Chimènes (éd.), op. cit., p. 99.

36 Lettre de Poulenc à Viñes, envoyée le [25 août 1917 de Paramé], dans M. Chimènes (éd.), op. cit., p. 54. Les douze conférences sont réunies dans un volume préfacé par Debussy, Pour la musique française, Paris-Zurich, Georges Crès et Cie, 1917.

37 Entretiens, op. cit., p. 24. 
dans la capitale française. Poulenc cherche à tout prix à se dégager de ces étiquettes quelque peu encombrantes et assure à Otto Marius Kling : «je ne suis pas un musicien Cubiste, encore moins Futuriste et bien entendu pas Impressionniste. Je suis un musicien sans étiquette ${ }^{38} »$.

CEuvre cyclique, déjà représentative de son auteur, les Mouvements perpétue/s connurent un succès jamais démenti. Nous l'avons dit, d'une part en raison des nombreuses adaptations dont elle bénéficia ${ }^{39}$, d'autre part en raison du nombre de concerts qui la programmèrent. Pour exemples (parmi bien d'autres), Milhaud les joue au Musikverein de Vienne le 7 février 1922, tandis que Poulenc les interprète dans un concert qui lui est consacré à la Salle du Conservatoire de Bruxelles le 17 janvier 1923 et Paul Collaer les joue aussi. On pourra ensuite les entendre en avril 1923, et au Maghreb (Oran, Alger, Tunis) en février 1935, soit un mois avant de les redécouvrir à l'Université des Annales, en mars 1935. Poulenc avoue plus tard aimer les entendre par Horowitz, plus que par Lucienne Delforge ${ }^{40}$. Premier chef-d'œuvre de Poulenc, le compositeur reconnaît entretenir une tendresse particulière pour cette œuvre et pour quelques autres plus tardives ${ }^{41}$. D'ailleurs, il grava ses Mouvements à New York chez Columbia (disque ML4399) avec une première face consacrée à Satie ${ }^{42}$. II

38 Lettre du 6 septembre 1919. C'est l'auteur qui souligne. Voir Myriam Chimènes (éd.), op. cit., p. 99.

39 Voir aussi la version pour piano et orchestre publiée chez Boosey et Hawkes en 1960.

40 Voir sa lettre de Noizay à André Jolivet [octobre 1943], dans Myriam Chimènes (éd.), op. cit., p. 543.

41 Interrogé sur les œuvres pour piano (de lui-même) qui trouvaient grâce à ses yeux, le compositeur répond: "C'est très simple, je tolère les Mouvements perpétuels, ma vieille Suite en ut, les Trois Pièces (anciennes Pastorales). J'aime beaucoup mes deux recueils d'Improvisations, un Intermezzo en lab et certains Nocturnes. Je condamne sans recours Napoli et les Soirées de Nazelles. Pour le reste, je ne m'en soucie guère »(Entretiens, op. cit., p. 35).

42 II en parle à Henri Sauguet dans une lettre du 12 février [1950], envoyée de Saint Moritz-On-The-Park, cf. Myriam Chimènes (éd.), op. cit., p. 675, ainsi qu'à Robert Douglas Gibson qui avait édité ses Mouvements en 1919 chez Chester (Londres). Voir la lettre de Chicago en date du 20 février [1950], id., p. 679. 
en fait lui-même la critique dans la revue des Arts phoniques 43 anticipant la réaction de certaines personnes qui s'étonneront des divergences de ses interprétations de concert avec celles de la version mécanique. Le compositeur portait donc un regard tout à fait objectif et clairvoyant sur ses compositions et ses interprétations, un regard introspectif et rétrospectif à la fois, Or, pour entreprendre réellement cette prise en compte d'un personnage dans sa globalité, il était important de revenir sur ces pièces de jeunesse, pleines de promesses, contenant déjà tout le Poulenc à venir. Et de cela, le compositeur en était bien conscient: "Je ne rougis pas d'être le musicien des Mouvements perpétuels ou du Bestiaire, bien au contraire, car ces deux œuvres sont très exactement représentatives du Poulenc de 1920 qu'a si merveilleusement dessiné Jean Cocteau ».

43 Francis Poulenc, "Musique instrumentale », Arts phoniques, no 10, novembredécembre 1928. Voir Myriam Chimènes (éd.), op. cit., p. 295. 


\section{ANNEXE 1}

Schéma formel du Ille Mouvement perpétue/ de Poulenc

\begin{tabular}{|c|c|c|c|c|c|}
\hline Mesures & $1-7$ & $8-36$ & $37-39$ & $40-50$ & $51-57$ \\
\hline Parties & A & B & $\begin{array}{c}A^{\prime} \\
\text { (tronqué) }\end{array}$ & C & $C^{\prime}$ \\
\hline Motifs & $\begin{array}{c}a_{1}+a^{\prime}{ }_{1} \\
(2+1 \\
\text { mes. }) \\
a_{2} \times 2+ \\
a_{3} \\
(1+1)+ \\
(2 \text { mes. })\end{array}$ & $\begin{array}{c}b_{1} \times 2+ \\
b_{2} \times 2 \\
(4+4 \\
\text { mes. }) \\
\\
b_{1} \times 2+ \\
b_{3} \\
(4+4 \\
\text { mes. }) \\
\\
b_{4}<b_{2} \\
\times 2 \\
(4+5 \\
\text { mes. })\end{array}$ & $\begin{array}{c}a_{1} \text { sans } \\
\text { anacrouse } \\
+a^{\prime}{ }_{1} \\
(2+1 \\
\text { mes.) }\end{array}$ & $\begin{array}{c}\mathrm{C}_{1} \text { en } \\
\text { symétrie } \\
(2+1+2 \\
\text { mes. }) \\
\mathrm{C}_{2} \\
(1+1 \\
\text { mes. }) \\
\mathrm{C}_{1} \\
(2+2 \\
\text { mes. }\end{array}$ & $\begin{array}{c}C^{\prime \prime}{ }_{1} \\
(2 \text { mes.) } \\
C_{2} \\
(1+1 \\
\text { mes.) } \\
\text { Reprise } 1 \text { e } \\
\text { fois } \\
\text { Reprise } \\
\text { dernière } \\
\text { (1 + } 1 \\
\text { mes.) } \\
+ \\
\text { Cadence } \\
\text { (1 mes.) }\end{array}$ \\
\hline $\begin{array}{l}\text { Chiffre } \\
\text { de } \\
\text { mesure }\end{array}$ & $\begin{array}{c}4 / 4+ \\
7 / 4 \\
4 / 4\end{array}$ & $\begin{array}{c}3 / 8 \\
\text { Croche }= \\
\text { croche }\end{array}$ & $4 / 4+7 / 4$ & $4 / 4$ & $\begin{array}{c}4 / 4 \\
5 / 4 \text { (aux } \\
\text { reprises) } \\
4 / 4\end{array}$ \\
\hline
\end{tabular}




\section{ANNEXE 2}

Schéma formel du Ile Mouvement perpétue/ de Poulenc

\begin{tabular}{|c|c|c|c|c|c|}
\hline Mesures & $1-2$ & $3-4$ & $5-10$ & $11-12$ & $13-14$ \\
\hline Motifs & $\begin{array}{c}a_{1}+a^{\prime}{ }_{1} \\
(1+1 \\
\text { mes. })\end{array}$ & $\begin{array}{c}a_{2}+a_{2}^{\prime} \\
(1+1 \\
\text { mes. })\end{array}$ & $\begin{array}{c}a_{3}+a_{3} \\
(1+1 \text { mes. } \\
a_{3}(m . d)+a_{4} \\
\quad(m . g) \\
a_{3}^{\prime}(m . d)+a_{4} \\
\quad(m . g) \\
a^{\prime \prime} 3(m . d)+ \\
a_{4} \times 2 \text { (m.g) } \\
(1+1+2 \\
\text { mes. })\end{array}$ & $\begin{array}{c}a_{5}+a_{5} \\
(1+1 \\
\text { mes. }) \\
+ \text { reprise }\end{array}$ & $\begin{array}{c}\mathrm{a}_{1}+ \\
\text { cadence } \\
(1+1 \\
\text { mes. })\end{array}$ \\
\hline $\begin{array}{c}\text { Chiffre de } \\
\text { mesure }\end{array}$ & $4 / 4$ & $4 / 4$ & $4 / 4$ & $6 / 4$ & $4 / 4$ \\
\hline
\end{tabular}

\section{ANNEXE 3}

Schéma formel du Jer Mouvement perpétue/ de Poulenc

\begin{tabular}{|c|c|c|c|c|c|}
\hline Mesures & $1-4$ & $5-7$ & $8-11$ & $12-19$ & $20-24$ \\
\hline Motifs & $\begin{array}{c}a_{1}+a_{1} \\
(2+2 \\
\text { mes. })\end{array}$ & $\begin{array}{c}b_{1}+b_{1}^{\prime} \\
(1+2 \\
\text { mes. })\end{array}$ & $\begin{array}{c}a_{1}+a_{2}=a_{1} \\
\text { transposé } \\
(2+2 \\
\text { mes. })\end{array}$ & $\begin{array}{c}\mathrm{c}+\mathrm{c} \\
(1+1 \\
\text { mes. }) \\
\mathrm{d}+\mathrm{d} \\
(2+2 \\
\text { mes. }) \\
+ \text { reprise }\end{array}$ & $\begin{array}{c}a_{1}+a_{3} \\
(2+2 \\
\text { mes. }) \\
+ \text { cadence } \\
\text { (1 mes.) }\end{array}$ \\
\hline
\end{tabular}




\section{Références}

BOUCHER, Maurice, Pour la musique française, Paris-Zurich, Georges Crès et Cie, 1917

CHIMENES, Myriam (éd.), Francis Poulenc, Correspondance (1910-1963), Paris, Fayard, 1994.

GEORGES, André, Les Nouvelles littéraires, 30 avril 1927.

KAYAS, Lucie, "Francis Poulenc disc jokey », dans Sidney Buckland et Myriam Chimènes (dir.), Francis Poulenc, Music, Art and Literature, Adelshot, Ashgate, 1999.

MILHAUD, Darius, Ma vie heureuse, Paris, Belfond, 1987.

POULENC, Francis, Entretiens avec Claude Rostand, Paris, R. Julliard, 1954.

RUNNING WARTON, Marjorie, "Poulenc and Dufy», dans Sidney Buckland et Myriam Chimènes (dir.), Francis Poulenc, Music, Art and Literature, Aldershot, Ashgate, 1999.

SCHMIDT, Carl B., The Music of Francis Poulenc (1899-1963). A Catalogue, Oxford, Clarendon Press, 1995. 